\title{
IV. GRAIN-SIZE AND CARBON/CARBONATE ANALYSES, LEG 43
}

\author{
Donald H. Cameron, Scripps Institution of Oceanography, La Jolla, California
}

\section{GRAIN-SIZE ANALYSES}

Sand-silt-clay distribution was determined at Scripps on samples collected at the time the cores were split and described. The results are listed in Table 1.

The sediment classification used here is that of Shepard (1954) with sand, silt, and clay boundaries based on the Wentworth (1922) scale. Thus the sand, silt, and clay fractions are composed of particles whose diameters range from 2000 to $62.5 \mu \mathrm{m}, 62.5$ to 3.91 $\mu \mathrm{m}$, and less than $3.91 \mu \mathrm{m}$, respectively. This classification is applied regardless of sediment type and origin; therefore, sediment names used in this table may differ from those used elsewhere in this volume; for example, a silt composed of nannofossils in this table may be called a nannofossil ooze in a site chapter.

Standard sieve and pipette methods were used to determine the grain-size distribution. The sediment sample was dried and dispersed in a Calgon solution. If a sediment sample failed to disaggregate, it was treated with a sonic probe and, if necessary, hydrogen peroxide. Sediment samples which resisted the above treatment were not analyzed.

The sand fraction was removed by wet sieving using a $62.5 \mu \mathrm{m}$ sieve, and the silt and clay fractions were analyzed by standard pipette analysis. Sampling depths and times were calculated using equations derived from Stokes settling velocity equation (Krumbein and Pettijohn, 1938, p. 95-96):

$$
\frac{\mathrm{D}}{\mathrm{t}}=\mathrm{V}=\frac{2 \mathrm{gr}^{2}\left(\mathrm{~d}_{1}-\mathrm{d}_{2}\right)}{9 \eta}
$$

where

$$
\begin{aligned}
\mathrm{V}= & \text { velocity, in cm/sec } \\
\mathrm{t}= & \text { time, in sec* } \\
\mathrm{D}= & \text { depth pipette is inserted, in } \mathrm{cm} \\
\mathrm{g}= & \text { gravity, in } \mathrm{cm} / \mathrm{sec}^{*} \\
\mathrm{r}= & \text { radius of individual particles, in cm* } \\
\mathrm{d}_{1}= & \text { density of solid particles arbitrarily set at } 2.65 \mathrm{~g} / \mathrm{cc} \\
\mathrm{d}_{2}= & \text { absolute density of distilled water at different tem- } \\
& \text { peratures (Hodgman et al., } 1960, \mathrm{p} .2129 \text { ). } \\
\eta= & \text { viscosity of distilled water in poises at different } \\
& \text { temperatures (Hodgman et al., 1960, p. } 2181)^{*}
\end{aligned}
$$

* Five figures were used in calculations to avoid rounding-off variations.
The reproducibility of the grain-size analyses has been previously tested (Boyce, 1972), and it was found that over a period of time with several operators the reproducibility for the sand-silt-clay fractions is \pm 2.5 per cent (absolute). For detailed step-by-step procedures, see Volume 4 of the Initial Reports of the Deep Sea Drilling Project.

\section{CARBON/CARBONATE ANALYSES}

Leg 43 sediments were analyzed for total carbon and acid-insoluble (organic) carbon using a LECO WR-12 Analyzer according to the standard technique outlined below. The 3-cc sediment samples were first dried at $105^{\circ}-110^{\circ} \mathrm{C}$ and then ground to a homogeneous powder. The ground sediment was redried and two samples, a $0.1 \mathrm{~g}$ and a $0.5 \mathrm{~g}$ sample, were then weighed into LECO clay crucibles. The 0.5 -g sample was acidified with a 10 per cent hydrochloric acid solution and then washed with distilled water. The sample was then dried and analyzed for acid-insoluble carbon, listed in Table 2 as "organic" carbon. The 0.1g sample was analyzed for total carbon without further treatment. If the result showed less than 10 per cent $\mathrm{CaCO}_{3}$, an additional 0.5 -g sample was analyzed for greater accuracy.

The calcium carbonate percentages were calculated

\begin{tabular}{|c|c|c|c|c|}
\hline $\begin{array}{l}\text { DSDP } \\
\text { Std. }\end{array}$ & $\begin{array}{c}\text { No. of } \\
\text { Samples }\end{array}$ & $\begin{array}{l}\text { Total Carbon } \\
\text { as } \% \mathrm{CaCO}_{3}\end{array}$ & $\begin{array}{l}\text { Standard } \\
\text { Deviation }\end{array}$ & $\begin{array}{c}\text { Maximum } \\
\text { Range }\end{array}$ \\
\hline 6 & $T_{T}$ & 92. & 2.5 & $5.8 \%$ \\
\hline 9 & 23 & 26.7 & $1.3 \%$ & $4.0 \%$ \\
\hline
\end{tabular}
as follows:

$$
(\% \text { total } \mathrm{C}-\% \text { organic } \mathrm{C}) \times 8.33=\% \mathrm{CaCO}_{3}
$$

Although other carbonates may be present, all acidsoluble carbon was calculated as calcium carbonate. All results are given in weight per cent (Table 2).

For control purposes standard sediments were made up from Deep Sea Drilling material and analyzed for total carbon at predetermined intervals with the regular samples. Listed below are the statistical data for these standards.

These data indicate the precision of the mechanical aspect of the LECO analysis and do not necessarily reflect the precision of the total analytical procedure, which may be affected by factors such as sampling techniques and contamination during sample preparation. 
Detailed descriptions of the technique and theory may be found in Bader, Gerard, et al. (1970) and Boyce and Bode (1972).

\section{REFERENCES}

Bader, R. G., Gerard, R. D., et al., 1970. Initial Reports of the Deep Sea Drilling Project, v. 4: Washington (U. S. Government Printing Office).

Boyce, R. E., 1972 Grain size analysis, Leg 9, Deep Sea Drilling Project. In Hays, J. D., et al., Initial Reports of the Deep Sea Drilling Project, v. 9: Washington (U. S. Government Printing Office), p. 779.
Boyce, R. E. and Bode, G. W., 1972. Carbon and carbonate analyses, Leg 9, Deep Sea Drilling Project. In Hays, J. D. et al., Initial Reports of the Deep Sea Drilling Project, v. 9: Washington (U. S. Government Printing Office), p. 747.

Hodgman, C. D., Weast, R. C., and Selby, S. M., 1960. Handbook of chemistry and physics: Cleveland (Chemical Rubber Publishing Co.), p. 3472.

Krumbein, W. C. and Pettijohn, F. J., 1938. Manual of sedimentary petrography: New York (Appleton-CenturyCrofts, Inc.).

Shepard, F. P., 1954. Nomenclature based on sand-silt-clay ratios: J. Sediment. Petrol., v. 24, p. 151.

Wentworth, C. K., 1922. A scale of grade and class terms for clastic sediments: J. Geol., v. 30, p. 377. 
1 ADLE 1

Grain-Size Analysis, DSDP LEG 43

\begin{tabular}{lcrrrl}
\hline $\begin{array}{c}\text { Sample } \\
\text { (Interval in cm) }\end{array}$ & $\begin{array}{c}\text { Depth } \\
(\mathrm{m})\end{array}$ & $\begin{array}{c}\text { Sand } \\
(\%)\end{array}$ & $\begin{array}{c}\text { Silt } \\
(\%)\end{array}$ & $\begin{array}{c}\text { Clay } \\
(\%)\end{array}$ & Classificatio \\
\hline Site 382 & & & & & \\
& & & & & \\
$6-4, \quad 15$ & 255.19 & 0.0 & 22.8 & 77.2 & Clay \\
$8-1,71$ & 279.75 & 0.0 & 15.1 & 84.9 & Clay \\
9-3, 68 & 291.88 & 0.0 & 18.3 & 81.7 & Clay \\
$10-2,60$ & 299.80 & 0.0 & 15.0 & 84.9 & Clay \\
$11-5,66$ & 314.46 & 0.0 & 16.7 & 83.2 & Clay \\
$12-4,59$ & 322.39 & 0.0 & 33.7 & 66.3 & Silty clay \\
$13-6,97$ & 335.26 & 0.0 & 18.7 & 81.3 & Clay \\
$14-5,35$ & 342.60 & 0.0 & 21.6 & 78.4 & Clay \\
$15-5,81$ & 352.69 & 0.0 & 5.9 & 94.1 & Clay \\
$16-5,94$ & 362.34 & 3.2 & 55.9 & 40.9 & Clayey silt \\
$17-3,34$ & 367.74 & 0.9 & 67.2 & 31.9 & Clayey silt \\
$18-1,108$ & 374.88 & 17.9 & 64.4 & 17.7 & Sandy silt \\
$18-2,36$ & 375.66 & 5.4 & 74.8 & 19.8 & Clayey silt \\
$18-4,137$ & 379.67 & 4.4 & 69.6 & 26.0 & Clayey silt \\
$20-2,114$ & 404.94 & 3.3 & 72.0 & 24.6 & Clayey silt \\
$21-2,80$ & 414.00 & 2.0 & 70.9 & 27.1 & Clayey silt \\
$21-3,123$ & 415.93 & 0.4 & 57.4 & 42.2 & Clayey silt \\
$22-1,122$ & 441.52 & 8.8 & 62.9 & 28.3 & Clayey silt
\end{tabular}

Site $\mathbf{3 8 3}$

$\begin{array}{rrrrrl}1-1,134 & 55.04 & 47.8 & 49.3 & 2.9 & \text { Sandy silt } \\ 1-2,115 & 56.35 & 87.8 & 10.4 & 1.8 & \text { Sand } \\ 1-3,110 & 57.80 & 94.4 & 4.5 & 1.0 & \text { Sand } \\ 1-4,17 & 58.37 & 96.3 & 2.8 & 1.0 & \text { Sand }\end{array}$

Site 384

\begin{tabular}{|c|c|c|c|c|c|}
\hline $2-3,120$ & 64.90 & 2.3 & 36.4 & 61.3 & Silty clay \\
\hline $4-1,130$ & 81.20 & 3.6 & 42.7 & 53.7 & Silty clay \\
\hline $6-3,142$ & 103.02 & 1.6 & 31.6 & 66.9 & Silty clay \\
\hline $7-3,130$ & 112. & 8.7 & 31.9 & 59.5 & Silty clay \\
\hline $8-2, \quad 77$ & 119.57 & 0.6 & 30.4 & 69.0 & Silty clay \\
\hline $9-3, \quad 98$ & 131.33 & 1.5 & 37.4 & 61.1 & Silty clay \\
\hline$-3,61$ & 140 & 6.5 & 35.4 & 58.1 & Silty clay \\
\hline 8 & 147. & 6.3 & 34.7 & 59.1 & Silty clay \\
\hline 63 & 15 & 8.7 & 35 & & Silty clay \\
\hline 36 & 166. & 8.8 & 32.0 & & Silty clay \\
\hline 81 & 177. & 3.7 & 31.8 & 64.4 & Silty cla \\
\hline 30 & 191.05 & 4.4 & 28.7 & 66.9 & Silty cl \\
\hline
\end{tabular}

Site 385

\begin{tabular}{llllll}
$1-2,89$ & 24.69 & 0.0 & 13.5 & 86.5 & Clay \\
$1-4,100$ & 27.80 & 2.0 & 20.5 & 77.6 & Clay \\
$2-2,120$ & 63.55 & 0.1 & 19.5 & 80.4 & Clay \\
$2-5,10$ & 66.95 & 0.0 & 25.6 & 74.3 & Silty clay \\
\hline
\end{tabular}

1 ADLE 1 - corlıriuea

\begin{tabular}{|c|c|c|c|c|c|}
\hline $\begin{array}{c}\text { Sample } \\
(\text { Interval in } \mathrm{cm})\end{array}$ & $\begin{array}{l}\text { Depth } \\
\text { (m) }\end{array}$ & $\underset{\%}{\text { Sand }}$ & $\begin{array}{l}\text { Silt } \\
\%\end{array}$ & $\begin{array}{c}\text { Clay } \\
\%\end{array}$ & Classification \\
\hline $3-2, \quad 90$ & 101.20 & 0.0 & 18.1 & 81.9 & Clay \\
\hline $3-5,100$ & 105.80 & 0.1 & 25.5 & 74.4 & Silty clay \\
\hline $4-2,100$ & 139.00 & 1.7 & 14.1 & 84.2 & Clay \\
\hline $5-2, \quad 30$ & 147.90 & 2.7 & 16.1 & 81.2 & Clay \\
\hline $5-4, \quad 50$ & 151.10 & 6.1 & 15.2 & 78.6 & Clay \\
\hline $8-5, \quad 88$ & 181.48 & 2.5 & 18.2 & 79.3 & Clay \\
\hline $9-1, \quad 94$ & 185.04 & 2.8 & 19.3 & 77.9 & Clay \\
\hline $10-1,112$ & 194.52 & 10.9 & 10.8 & 78.3 & Clay \\
\hline $11-1,143$ & 204.43 & 0.4 & 22.4 & 77.2 & Clay \\
\hline $12-2,115$ & 215.15 & 0.0 & 14.9 & 85.0 & Clay \\
\hline $13-2,90$ & 234.00 & 0.0 & 12.6 & 87.4 & Clay \\
\hline
\end{tabular}

Site 386

\begin{tabular}{|c|c|c|c|c|c|}
\hline $1-2,120$ & 55.30 & 2.1 & 32.2 & 65.7 & Silty clay \\
\hline $1-5,100$ & 59.60 & 5.8 & 17.6 & 76.6 & Clay \\
\hline $3-1,138$ & 139.38 & 0.2 & 7.7 & 92.2 & Clay \\
\hline $4-1,106$ & 148.26 & 7.0 & 36.4 & 56.5 & Silty clay \\
\hline $4-2,125$ & 149.95 & 0.1 & 6.2 & 93.7 & Clay \\
\hline $4-6, \quad 82$ & 155.52 & 41.3 & 33.7 & 25.0 & Sand-silt-clay \\
\hline $5-2$ & 158.36 & 0.1 & 50.8 & 49.2 & Clayey silt \\
\hline $5-2,102$ & 159.32 & 83.2 & 12.2 & 4.6 & Sand \\
\hline $6-1,99$ & 167.29 & 67.8 & 25.6 & 6.7 & Silty sand \\
\hline $6-2,130$ & 169.10 & 0.0 & 52.2 & 47.7 & Clayey silt \\
\hline $6-3,64$ & 169.94 & 0.1 & 56.8 & 43.2 & Clayey silt \\
\hline $7-1,139$ & 177.19 & 33.8 & 37.6 & 28.6 & Sand-silt-clay \\
\hline $8-5,101$ & 192.31 & 60.3 & 18.9 & 20.8 & Clayey sand \\
\hline $9-2$ & 205.87 & 35.1 & 33.9 & 31.0 & Sand-silt-clay \\
\hline $12-2, \quad 69$ & 282.59 & 2.6 & 41.2 & 56.2 & Silty clay \\
\hline 15-2, & 348.95 & 12.8 & 56.1 & 31.1 & Clayey silt \\
\hline $15-5,49$ & 353.89 & 0.3 & 29.5 & 70.2 & Silty clay \\
\hline $16-5,107$ & 373.07 & 3.1 & 38.7 & 58.1 & Silty clay \\
\hline $17-2, \quad 86$ & 387.86 & 3.6 & 66.4 & 30.0 & Clayey silt \\
\hline $19-2, \quad 81$ & 415.91 & 0.0 & 39.1 & 60.9 & Silty clay \\
\hline $28-2,116$ & 502.06 & 0.0 & 20.0 & 80.0 & Clay \\
\hline $30-6, \quad 89$ & 526.79 & 0.1 & 19.0 & 80.9 & Clay \\
\hline $32-5, \quad 10$ & 562.65 & 0.1 & 18.9 & 81.0 & Clay \\
\hline
\end{tabular}

Site 387

\begin{tabular}{rrrrrl}
$1-2,65$ & 33.95 & 0.0 & 3.6 & 96.3 & Clay \\
$6-3,49$ & 168.59 & 9.5 & 22.4 & 68.1 & Silty clay \\
$7-5,70$ & 181.65 & 2.8 & 30.5 & 66.7 & Silty clay \\
$8-1,90$ & 185.10 & 2.3 & 38.9 & 58.8 & Silty clay \\
$10-5,77$ & 210.37 & 3.8 & 37.6 & 58.6 & Silty clay \\
$11-1,145$ & 214.25 & 0.0 & 42.5 & 57.5 & Silty clay \\
$27-6,123$ & 449.98 & 0.0 & 37.0 & 62.9 & Silty clay \\
$29-2,85$ & 471.75 & 0.1 & 18.7 & 81.2 & Clay \\
$37-2,62$ & 576.42 & 0.3 & 19.5 & 80.3 & Clay \\
$37-2,122$ & 577.02 & 3.9 & 11.7 & 84.4 & Clay \\
\hline
\end{tabular}


TABLE 2

TABLE 2 - Continued

Carbon/Carbonate Analyses, DSDP LEG 43

\begin{tabular}{ccccc}
\hline $\begin{array}{c}\text { Sample } \\
\text { (Interval in cm) }\end{array}$ & $\begin{array}{c}\text { Depth } \\
(\mathrm{m})\end{array}$ & $\begin{array}{c}\text { Total } \\
\text { Carbon }\end{array}$ & $\begin{array}{c}\text { Organic } \\
\text { Carbon }\end{array}$ & $\mathrm{CaCO}_{3}$ \\
\hline
\end{tabular}

\section{Site 382}

$\begin{array}{rrrrr}1-1,50 & 51.5 & 1.5 & 0.4 & 10 \\ 16-2,4 & 356.9 & 1.1 & 0.0 & 9 \\ 17-4,116 & 370.1 & 0.3 & 0.0 & 2 \\ 20-5,148 & 409.8 & 0.1 & 0.0 & 0\end{array}$

Site 384

\begin{tabular}{|c|c|c|c|c|}
\hline $1-3,80$ & 54.6 & 6.3 & 0.1 & 52 \\
\hline $2-1,90$ & 61.6 & 5.7 & 0.0 & 47 \\
\hline $2-2, \quad 50$ & 62.7 & 6.2 & 0.1 & 51 \\
\hline $2-4, \quad 50$ & 65.7 & 6.5 & 0.0 & 54 \\
\hline $2-5,100$ & 67.7 & 6.3 & 0.0 & 52 \\
\hline $2-6, \quad 80$ & 69.0 & 6.7 & 0.0 & 56 \\
\hline $3-1, \quad 50$ & 70.5 & 6.9 & 0.1 & 57 \\
\hline $4-1, \quad 60$ & 80.5 & 6.8 & 0.0 & 56 \\
\hline $4-2,100$ & 82.4 & 6.3 & 0.0 & 52 \\
\hline $4-4,100$ & 85.4 & 6.6 & 0.0 & 55 \\
\hline $4-5, \quad 90$ & 86.8 & 7.5 & 0.0 & 62 \\
\hline $4-6,139$ & 88.8 & 8.3 & 0.0 & 69 \\
\hline $5-1,120$ & 90.2 & 9.5 & 0.0 & 79 \\
\hline $6-1,118$ & 99.8 & 8.9 & 0.0 & 74 \\
\hline $6-3,10$ & 101.7 & 8.8 & 0.0 & 73 \\
\hline $7-3,88$ & 111.9 & 9.5 & 0.0 & 79 \\
\hline $7-6, \quad 96$ & 116.5 & 8.6 & 0.0 & 72 \\
\hline $8-2, \quad 25$ & 119.1 & 9.2 & 0.0 & 76 \\
\hline $8-5,46$ & 123.8 & 9.2 & 0.0 & 76 \\
\hline $9-3,116$ & 131.5 & 7.0 & 0.0 & 58 \\
\hline $9-5,82$ & 134.2 & 8.4 & 0.0 & 70 \\
\hline $10-2, \quad 61$ & 138.5 & 8.2 & 0.0 & 68 \\
\hline $10-3, \quad 75$ & 140.2 & 9.1 & 0.0 & 75 \\
\hline $10-6,140$ & 145.3 & 8.3 & 0.0 & 69 \\
\hline $11-2, \quad 71$ & 147.9 & 7.7 & 0.0 & 64 \\
\hline $11-4, \quad 16$ & 150.4 & 8.4 & 0.0 & 70 \\
\hline $12-2, \quad 39$ & 156.9 & 8.9 & 0.0 & 74 \\
\hline 12-5, & 161.1 & 9.1 & 0.0 & 76 \\
\hline $13-2, \quad 62$ & 166.7 & 10.3 & 0.0 & 85 \\
\hline $13-4, \quad 18$ & 169.3 & 10.8 & 0.0 & 90 \\
\hline $13-5, \quad 75$ & 171.4 & 10.7 & 0.0 & 89 \\
\hline $14-2, \quad 60$ & 175.7 & 11.0 & 0.0 & 91 \\
\hline $15-1, \quad 66$ & 183.9 & 11.0 & 0.0 & 91 \\
\hline $15-6,125$ & 192.0 & 10.5 & 0.0 & 87 \\
\hline
\end{tabular}

Site 385

\begin{tabular}{rrrrr}
$1-2, \quad 50$ & 24.3 & 0.2 & 0.2 & 0 \\
$1-4,75$ & 27.6 & 0.1 & 0.1 & 0 \\
$2-2,80$ & 63.2 & 0.2 & 0.2 & 0 \\
$3-2,100$ & 101.3 & 0.2 & 0.2 & 0 \\
$3-5,75$ & 105.6 & 0.3 & 0.2 & 0 \\
$4-2,68$ & 138.7 & 0.1 & 0.1 & 0 \\
$5-2,80$ & 148.4 & 0.1 & 0.1 & 0 \\
$8-5,113$ & 181.7 & 0.1 & 0.1 & 0 \\
$9-1,115$ & 185.3 & 0.0 & 0.1 & 0 \\
$10-1,133$ & 194.7 & 0.1 & 0.1 & 0 \\
$11-2,70$ & 205.2 & 0.3 & 0.1 & 2 \\
$12-2,70$ & 214.7 & 3.4 & 0.1 & 28 \\
$13-2,95$ & 234.1 & 5.4 & 0.1 & 44 \\
$13-4,77$ & 236.9 & 6.1 & 0.9 & 44 \\
$14-2,84$ & 252.9 & 0.1 & 0.1 & 0 \\
$15-1,80$ & 270.4 & 0.1 & 0.1 & 0 \\
\hline
\end{tabular}

\begin{tabular}{ccccc}
\hline $\begin{array}{c}\text { Sample } \\
\text { (Interval in cm) }\end{array}$ & $\begin{array}{c}\text { Depth } \\
(\mathrm{m})\end{array}$ & $\begin{array}{c}\text { Total } \\
\text { Carbon }\end{array}$ & $\begin{array}{l}\text { Organic } \\
\text { Carbon }\end{array}$ & $\mathrm{CaCO}_{3}$ \\
\hline
\end{tabular}

Site 386

\begin{tabular}{|c|c|c|c|c|}
\hline $1-2, \quad 70$ & 54.8 & 2.0 & 0.2 & 15 \\
\hline $1-5,80$ & 59.4 & 8.2 & 0.1 & 68 \\
\hline $1-6,80$ & 60.9 & 0.1 & 0.1 & 0 \\
\hline $2-5, \quad 91$ & 107.0 & 0.3 & 0.2 & 0 \\
\hline $3-1,118$ & 139.2 & 0.9 & 0.2 & 6 \\
\hline $4-1,120$ & 148.4 & 0.1 & 0.1 & 0 \\
\hline $4-2, \quad 80$ & 149.5 & 0.1 & 0.1 & 0 \\
\hline $4-4, \quad 80$ & 152.5 & 3.9 & 0.1 & 32 \\
\hline $5-2,130$ & 159.6 & 0.1 & 0.0 & 0 \\
\hline $5-4,90$ & 162.2 & 0.1 & 0.1 & 0 \\
\hline $6-1,101$ & 167.3 & 1.0 & 0.0 & 8 \\
\hline $6-3, \quad 70$ & 170.0 & 2.3 & 0.0 & 19 \\
\hline $8-1,100$ & 186.3 & 1.9 & 0.1 & 15 \\
\hline $9-2,33$ & 206.1 & 1.4 & 0.1 & 12 \\
\hline $11-2, \quad 73$ & 254.1 & 2.4 & 0.1 & 19 \\
\hline $11-3,113$ & 256.0 & 3.5 & 0.1 & 29 \\
\hline $12-2,103$ & 282.9 & 2.7 & 0.1 & 22 \\
\hline $13-3, \quad 69$ & 312.7 & 0.2 & 0.0 & 1 \\
\hline $14-2, \quad 25$ & 330.3 & 2.1 & 0.3 & 15 \\
\hline $14-6,135$ & 337.4 & 3.2 & 0.3 & 25 \\
\hline $15-2,121$ & 350.1 & 3.4 & 0.2 & 27 \\
\hline $15-5, \quad 89$ & 354.3 & 3.3 & 0.1 & 26 \\
\hline $16-3, \quad 34$ & 369.3 & 1.2 & 0.3 & 8 \\
\hline $16-3, \quad 46$ & 369.5 & 3.1 & 0.1 & 25 \\
\hline $17-3, \quad 61$ & 389.1 & 1.3 & 0.7 & 5 \\
\hline $17-5, \quad 51$ & 392.0 & 1.9 & 0.5 & 12 \\
\hline $18-1, \quad 27$ & 404.4 & 1.5 & 0.7 & 7 \\
\hline $19-2, \quad 88$ & 416.0 & 2.9 & 0.2 & 23 \\
\hline $20-3,122$ & 427.3 & 3.1 & 0.5 & 22 \\
\hline $21-2,123$ & 435.4 & 1.6 & 0.3 & 10 \\
\hline $21-5, \quad 9$ & 438.8 & 10.2 & 0.1 & 84 \\
\hline $22-1,110$ & 443.2 & 2.4 & 1.0 & 12 \\
\hline $22-4,105$ & 447.7 & 3.3 & 0.4 & 24 \\
\hline $23-2,141$ & 454.6 & 3.6 & 0.5 & 26 \\
\hline $23-4, \quad 15$ & 456.4 & 1.9 & 0.6 & 11 \\
\hline $24-2,118$ & 463.9 & 3.7 & 0.4 & 27 \\
\hline $24-4, \quad 69$ & 466.4 & 5.9 & 0.1 & 49 \\
\hline $25-1, \quad 51$ & 471.8 & 0.4 & 0.2 & 2 \\
\hline $25-5, \quad 2$ & 477.3 & 1.7 & 0.8 & 8 \\
\hline $26-2,131$ & 483.1 & 3.2 & 0.3 & 24 \\
\hline $26-5,87$ & 487.2 & 0.8 & 0.7 & 1 \\
\hline $27-2, \quad 60$ & 491.9 & 1.5 & 0.6 & 7 \\
\hline $27-4, \quad 30$ & 494.6 & 0.8 & 0.4 & 3 \\
\hline $28-2, \quad 79$ & 501.7 & 3.6 & 0.1 & 29 \\
\hline $29-2, \quad 60$ & 511.0 & 4.0 & 0.2 & 32 \\
\hline $30-2, \quad 20$ & 520.1 & 1.1 & 0.7 & 3 \\
\hline $31-1, \quad 30$ & 538.1 & 0.1 & 0.1 & 0 \\
\hline $31-6, \quad 80$ & 546.1 & 2.1 & 0.3 & 16 \\
\hline $32-2,119$ & 559.2 & 2.4 & 0.1 & 19 \\
\hline $32-5, \quad 52$ & 563.1 & 0.1 & 0.1 & 0 \\
\hline $32-5,149$ & 564.0 & 2.5 & 0.2 & 20 \\
\hline $33-2, \quad 67$ & 577.4 & 3.3 & 0.1 & 27 \\
\hline $34-2, \quad 88$ & 606.5 & 1.6 & 0.1 & 12 \\
\hline $34-6, \quad 23$ & 611.9 & 0.7 & 0.1 & 5 \\
\hline $35-4,46$ & 637.0 & 7.6 & 0.1 & 63 \\
\hline $35-5,119$ & 639.2 & 0.1 & 0.1 & 0 \\
\hline $36-3, \quad 58$ & 645.1 & 0.2 & 0.1 & 1 \\
\hline $36-5, \quad 56$ & 648.1 & 0.3 & 0.1 & 2 \\
\hline 38-3, & 692.5 & 0.1 & 0.0 & 0 \\
\hline 38-5, & 695.5 & 0.1 & 0.0 & 1 \\
\hline
\end{tabular}


TABLE 2 - Continued

\begin{tabular}{|c|c|c|c|c|}
\hline $\begin{array}{c}\text { Sample } \\
\text { (Interval in cm) }\end{array}$ & $\begin{array}{l}\text { Depth } \\
\text { (m) }\end{array}$ & $\begin{array}{c}\text { Total } \\
\text { Carbon }\end{array}$ & $\begin{array}{l}\text { Organic } \\
\text { Carbon }\end{array}$ & $\mathrm{CaCO}_{3}$ \\
\hline $39-1, \quad 11$ & 699.1 & 0.1 & 0.0 & 0 \\
\hline $39-6, \quad 7$ & 706.5 & 0.1 & 0.0 & 0 \\
\hline $39-6, \quad 30$ & 706.8 & 0.1 & 0.1 & 1 \\
\hline $40-3,130$ & 712.3 & 0.1 & 0.0 & 1 \\
\hline $41-1,119$ & 718.7 & 0.0 & 0.0 & 0 \\
\hline $41-5, \quad 78$ & 724.3 & 0.1 & 0.1 & 0 \\
\hline $42-4, \quad 54$ & 732.0 & 0.1 & 0.1 & 0 \\
\hline $43-2, \quad 14$ & 737.9 & 0.2 & 0.1 & 1 \\
\hline $43-2,135$ & 739.2 & 13.5 & 11.5 & 17 \\
\hline $44-2, \quad 82$ & 748.1 & 2.1 & 2.0 & 1 \\
\hline $44-3,126$ & 750.1 & 1.6 & 0.2 & 12 \\
\hline $45-1, \quad 63$ & 766.0 & 4.0 & 1.9 & 17 \\
\hline $45-1,100$ & 766.4 & 0.2 & 0.1 & 1 \\
\hline $45-2,102$ & 767.9 & 8.2 & 1.9 & 52 \\
\hline $45-6, \quad 93$ & 773.8 & 0.1 & 0.1 & 0 \\
\hline $46-2, \quad 42$ & 776.3 & 0.1 & 0.1 & 0 \\
\hline $46-5,53$ & 780.9 & 0.2 & 0.2 & 0 \\
\hline $47-3, \quad 88$ & 787.7 & 0.1 & 0.1 & 0 \\
\hline $47-4, \quad 58$ & 788.9 & 0.7 & 0.6 & 1 \\
\hline $48-2,145$ & 796.3 & 5.9 & 0.5 & 45 \\
\hline $49-3,145$ & 807.3 & 1.8 & 0.8 & 8 \\
\hline $49-4, \quad 66$ & 808.0 & 4.1 & 0.2 & 33 \\
\hline $50-3, \quad 31$ & 815.6 & 3.4 & 1.6 & 15 \\
\hline $50-5,134$ & 819.6 & 9.6 & 2.8 & 57 \\
\hline $51-1,105$ & 823.4 & 4.0 & 1.7 & 20 \\
\hline $51-5, \quad 57$ & 828.9 & 10.3 & 2.4 & 66 \\
\hline $52-2, \quad 83$ & 833.7 & 5.3 & 1.8 & 29 \\
\hline $52-6,100$ & 839.9 & 5.5 & 1.5 & 33 \\
\hline $53-2,67$ & 843.1 & 8.0 & 0.7 & 61 \\
\hline $53-4, \quad 59$ & 846.0 & 0.9 & 0.6 & 2 \\
\hline $54-3, \quad 24$ & 853.9 & 4.2 & 1.3 & 25 \\
\hline $54-3,86$ & 854.5 & 0.9 & 0.4 & 4 \\
\hline $55-1, \quad 92$ & 860.8 & 2.2 & 1.0 & 9 \\
\hline $55-6, \quad 49$ & 867.9 & 8.9 & 2.2 & 56 \\
\hline $56-3,47$ & 872.8 & 6.8 & 1.7 & 43 \\
\hline $56-4,121$ & 875.0 & 3.7 & 1.7 & 16 \\
\hline $57-1,53$ & 879.4 & 2.3 & 1.3 & 8 \\
\hline $57-6,94$ & 887.3 & 6.3 & 1.2 & 43 \\
\hline $58-1, \quad 85$ & 889.4 & 3.0 & 0.3 & 23 \\
\hline $58-2, \quad 65$ & 890.7 & 6.4 & 1.9 & 38 \\
\hline $59-3, \quad 98$ & 901.7 & 6.7 & 1.4 & 44 \\
\hline $59-5, \quad 49$ & 904.2 & 6.0 & 2.2 & 32 \\
\hline $60-2,127$ & 910.0 & 0.5 & 0.5 & 0 \\
\hline $60-4, \quad 23$ & 911.9 & 8.1 & 4.0 & 34 \\
\hline $61-2, \quad 56$ & 918.9 & 1.1 & 1.1 & 0 \\
\hline $61-4, \quad 65$ & 921.9 & 0.4 & 0.3 & 0 \\
\hline $62-2,117$ & 929.0 & 1.4 & 1.2 & 2 \\
\hline $62-6, \quad 54$ & 934.3 & 3.0 & 2.9 & 1 \\
\hline $63-3,114$ & 939.9 & 1.8 & 1.8 & 0 \\
\hline $63-4,140$ & 941.7 & 0.1 & 0.1 & 0 \\
\hline $64-2,108$ & 947.9 & 0.1 & 0.1 & 0 \\
\hline $64-4, \quad 61$ & 950.4 & 1.5 & 1.4 & 1 \\
\hline $65-1,81$ & 955.6 & 3.6 & 3.5 & 1 \\
\hline $65-2,60$ & 956.9 & 0.2 & 0.2 & 0 \\
\hline \multicolumn{5}{|l|}{ Site 387} \\
\hline $1-2, \quad 85$ & 34.2 & 0.1 & 0.1 & 0 \\
\hline $1-6,117$ & 40.5 & 0.2 & 0.1 & 0 \\
\hline $2-2,100$ & 101.0 & 0.2 & 0.1 & 0 \\
\hline $2-5, \quad 60$ & 105.1 & 0.1 & 0.1 & 0 \\
\hline
\end{tabular}

TABLE 2 - Continued

\begin{tabular}{|c|c|c|c|c|}
\hline $\begin{array}{c}\text { Sample } \\
\text { (Interval in } \mathrm{cm} \text { ) }\end{array}$ & $\begin{array}{l}\text { Depth } \\
(\mathrm{m})\end{array}$ & $\begin{array}{l}\text { Total } \\
\text { Carbon }\end{array}$ & $\begin{array}{l}\text { Organic } \\
\text { Carbon }\end{array}$ & $\mathrm{CaCO}_{3}$ \\
\hline $3-2,110$ & 139.2 & 0.1 & 0.1 & 0 \\
\hline $4-1, \quad 70$ & 146.8 & 0.2 & 0.1 & 1 \\
\hline $5, \mathrm{CC}, 10$ & 155.7 & 0.2 & 0.1 & 1 \\
\hline $6-3,90$ & 169.0 & 0.2 & 0.1 & 1 \\
\hline $7-1,90$ & 175.9 & 0.3 & 0.3 & 0 \\
\hline $7-5,90$ & 181.9 & 0.2 & 0.1 & 1 \\
\hline $8-1, \quad 70$ & 184.9 & 0.2 & 0.1 & 1 \\
\hline $8-2, \quad 9$ & 185.8 & 2.8 & 0.2 & 21 \\
\hline $9-2, \quad 40$ & 196.0 & 0.1 & 0.1 & 0 \\
\hline $9-6, \quad 40$ & 202.0 & 2.5 & 0.1 & 19 \\
\hline $10-4,130$ & 209.4 & 0.9 & 0.2 & 6 \\
\hline $10-5, \quad 70$ & 210.3 & 1.5 & 0.1 & 12 \\
\hline $11-1,135$ & 214.1 & 0.1 & 0.1 & 0 \\
\hline $12-1,132$ & 223.6 & 0.2 & 0.2 & 0 \\
\hline $13-1, \quad 74$ & 232.5 & 2.1 & 0.6 & 13 \\
\hline $14-2,42$ & 243.2 & 1.1 & 0.2 & 7 \\
\hline $16-1,139$ & 261.5 & 1.4 & 0.1 & 10 \\
\hline $17-1,95$ & 270.6 & 0.5 & 0.4 & 1 \\
\hline $18-1, \quad 32$ & 289.0 & 0.1 & 0.1 & 0 \\
\hline $19-2, \quad 65$ & 309.9 & 0.5 & 0.1 & 3 \\
\hline $19-3,111$ & 311.8 & 0.8 & 0.1 & 6 \\
\hline $20-1,132$ & 328.0 & 0.6 & 0.2 & 4 \\
\hline $20-3, \quad 65$ & 330.4 & 2.1 & 0.2 & 15 \\
\hline $21-1,128$ & 347.0 & 3.2 & 0.1 & 26 \\
\hline $21-2, \quad 89$ & 348.1 & 1.6 & 0.5 & 9 \\
\hline $22-2, \quad 29$ & 357.0 & 2.2 & 0.3 & 16 \\
\hline $22-2, \quad 79$ & 357.5 & 2.5 & 0.1 & 20 \\
\hline $23-1, \quad 29$ & 374.6 & 3.6 & 0.1 & 30 \\
\hline $24-1,140$ & 394.7 & 2.5 & 1.3 & 9 \\
\hline $25-3, \quad 73$ & 406.5 & 0.4 & 0.1 & 2 \\
\hline $26-2, \quad 21$ & 413.9 & 0.2 & 0.1 & 0 \\
\hline $27-1, \quad 64$ & 441.9 & 1.0 & 1.0 & 0 \\
\hline $28-1,116$ & 461.1 & 5.6 & 0.7 & 41 \\
\hline $29-2, \quad 71$ & 471.6 & 4.2 & 0.1 & 34 \\
\hline $29-3, \quad 88$ & 473.3 & 0.1 & 0.0 & 0 \\
\hline $30-1,136$ & 489.9 & 13.1 & 11.3 & 15 \\
\hline $31-1,138$ & 508.9 & 0.1 & 0.1 & 0 \\
\hline $32-1,111$ & 518.6 & 0.1 & 0.1 & 0 \\
\hline $32-2, \quad 79$ & 519.8 & 0.7 & 0.7 & 0 \\
\hline $33-2, \quad 60$ & 528.7 & 0.5 & 0.5 & 0 \\
\hline $34-2, \quad 71$ & 538.4 & 0.5 & 0.5 & 0 \\
\hline $34-4, \quad 33$ & 541.0 & 0.3 & 0.3 & 0 \\
\hline $35-3, \quad 69$ & 549.4 & 2.9 & 2.8 & 1 \\
\hline $35-5,90$ & 552.6 & 0.1 & 0.1 & 0 \\
\hline $36-1, \quad 80$ & 556.1 & 0.1 & 0.1 & 0 \\
\hline $36-3,80$ & 559.1 & 4.2 & 3.7 & 5 \\
\hline $37-2,127$ & 577.1 & 0.2 & 0.2 & 0 \\
\hline $38-1, \quad 65$ & 593.8 & 11.4 & 0.9 & 88 \\
\hline $39-2, \quad 68$ & 623.8 & 11.0 & 3.1 & 66 \\
\hline $39-2,113$ & 624.2 & 10.3 & 0.4 & 82 \\
\hline $40-1, \quad 90$ & 632.0 & 9.6 & 2.6 & 59 \\
\hline $41-1, \quad 68$ & 641.3 & 8.8 & 3.0 & 48 \\
\hline $42-1, \quad 93$ & 651.1 & 11.0 & 4.8 & 51 \\
\hline $44-1, \quad 76$ & 679.5 & 9.5 & 3.8 & 48 \\
\hline $45-1,125$ & 699.0 & 11.0 & 1.5 & 79 \\
\hline $46-1,125$ & 727.3 & 11.5 & 0.3 & 94 \\
\hline $46-2, \quad 56$ & 728.1 & 11.4 & 0.0 & 94 \\
\hline $47-1,140$ & 746.3 & 10.3 & 1.6 & 72 \\
\hline $48-1, \quad 85$ & 764.6 & 10.9 & 0.7 & 85 \\
\hline $49-2, \quad 89$ & 784.8 & 8.4 & 1.5 & 58 \\
\hline $49-5,111$ & 789.5 & 8.0 & 1.1 & 57 \\
\hline
\end{tabular}

